\title{
Determination of the instantaneous forces on flapping wings from a localized fluid velocity field
}

\author{
F. O. Minotti \\ Departamento de Física, Instituto de Física del Plasma, INFIP-CONICET, Universidad de Buenos Aires, \\ C1428EHA Buenos Aires, Argentina
}

(Received 4 August 2011; accepted 20 October 2011; published online 10 November 2011)

\begin{abstract}
Expressions are derived to relate the instantaneous pressure force on a flapping wing to the velocity field on a plane at the trailing edge and on a highly localized region around and near the wing, valid when the vortex sheet is thin. In its more practical version, the formalism is applicable to wings with close to two-dimensional geometry and has the advantage of not using spatial derivatives, but only a time derivative of a surface integral of the velocity. In the purely two-dimensional case, the expression obtained is used to justify a much simpler one that only requires the evaluation of the time derivative of the wing circulation. A comparison with a numerical simulation in a two-dimensional case shows a good representation of the forces, even with the most simplified expression, when the condition of a thin wake is met. Other examples are shown in which the wake is not thin in order to explore the limitations of the formalism. It is found in these cases that the thrust is sometimes not so well reproduced, with a tendency to be overestimated, while the lift is generally better reproduced. Remarkably, the simpler expression reproduces rather acceptably the phase and amplitude of both thrust and lift in all cases. (C) 2011 American Institute of Physics. [doi:10.1063/1.3659496]
\end{abstract}

There is a continuously growing interest in the study of flapping propulsion of swimming and flying animals, ${ }^{1,2}$ which is motivated by the natural fascination that this subject provokes, as well as by possible applications to mechanical devices. Of great interest is the direct measurement of the forces on flapping wings or fins, either of the animal itself or of mechanical models thereof. Naturally, this is particularly difficult in the former case and some way of obtaining these forces without interfering with the animal motion is highly desirable. A very attractive possibility is to derive the forces from the fluid velocity flow generated by the flapping itself. This is in principle possible by the conservation of fluid momentum if the velocity field could be known in rather large regions around the animal. For instance, the vorticity impulse formulation given in Refs. 3 and 4 allows to evaluate forces basically from the time derivative of a moment of the distribution of fluid vorticity. Comparison of related approaches can be found in the comprehensive work of Noca et al..$^{5}$ In particular, if the fluid velocity, its spatial derivatives, and the acceleration are known on a control surface surrounding the body, the formulation in Ref. 6 allows to determine instantaneous forces as well. On the other hand, in the case of periodic flapping, in the limit of inviscid, incompressible flow with generated vorticity limited to a thin wake, the average of the force in one flapping period can be obtained from the knowledge of the velocity field in a rather restricted region, known as the Trefftz volume. ${ }^{7}$ The great advance in optical velocimetry $^{8,9}$ thus makes it possible to obtain instantaneous or averaged forces in a non-intrusive way. The objective of this work is to show that if the vorticity is shed in the form of a thin sheet (at least close to the flapping appendage), the instantaneous pressure force can be determined from the velocity field in a rather restricted region, notably, more limited than the Trefftz volume. An additional advantage is that the only time derivative needed is that of a global magnitude determined from the velocity field close to the appendage and no spatial derivatives of the velocity field are needed, which is very convenient from a practical point of view. ${ }^{10}$ The main limitation is that the shed vorticity has to be limited to a thin sheet near the trailing edge (the subsequent rolling up of this sheet, with the formation of complex vortical structures, has no consequence as long as one considers the flow in the region near the extremity). This is the case in general for forward swimming or flight with streamlined appendages, as considered in Ref. 7, but not, for instance, for the pectoral fin beat of fishes ${ }^{11}$ or the hovering of birds. ${ }^{12}$ Also, as the fluid is considered outside the boundary layers, due to the assumption of potential flow, only the pressure contribution to the force is evaluated, which should be an accurate estimation of the total force at high enough Reynolds numbers.

Following Ref. 7, we consider the flapping extremity (wing to be concise) moving in a fluid at rest far away. At variance with Ref. 7 , we will not split the wing velocity in the average velocity of the animal plus a perturbation, which amounts to taking $U=0$ in the expressions given by Ref. 7 . In this way, the force on the wing $\mathbf{F}(t)$ can be expressed as (Eq. (A5) in the Appendix of Ref. 7).

$$
\begin{aligned}
\mathbf{F}(t)= & -\frac{d}{d t} \int_{C V} \rho \mathbf{u} d^{3} x-\int_{S_{T}} \rho(v \mathbf{j}+w \mathbf{k}) u d y d z \\
& +\int_{S_{T}} \frac{1}{2} \rho\left(2 \frac{\partial \phi}{\partial t}-u^{2}+v^{2}+w^{2}\right) \mathbf{i} d y d z
\end{aligned}
$$

In this expression, $\mathbf{u}$ is the fluid velocity given by

$$
\mathbf{u}=\nabla \phi=u \mathbf{i}+v \mathbf{j}+w \mathbf{k}
$$


and $\rho$ is the constant fluid density. The volume integral is performed in the control volume $C V$ that encloses the wing, limited in the wake region by the Trefftz plane $S_{T}$, normal to the average direction of flight and stationary, and at a finite distance from the trailing edge. $C V$ extends to infinity in the other directions and is limited internally by the wing surface (see Fig. 1). Note also that the wake is assumed thin at all places inside the control volume, so that the Trefftz plane is to be placed close enough to the wing so that no rolling up of the vorticity sheet happens inside that volume. In the same line, the effect of wing tip vortices is not taken into account, so that the formalism is applicable to wings of large enough aspect ratio.

It is worth mentioning that if one considers that the fluid far away from the wing has a uniform and constant velocity $\mathbf{U}_{\infty}$, a direct derivation along the same lines as in Ref. 7 shows that the force on the body is in that case given by Eq. (1), with the additional term

$$
\int_{S_{T}} \rho\left[\left(\mathbf{u} \cdot \mathbf{U}_{\infty}\right) \mathbf{i}-\mathbf{u}\left(\mathbf{U}_{\infty} \cdot \mathbf{i}\right)-\mathbf{U}_{\infty}(\mathbf{u} \cdot \mathbf{i})\right] d y d z .
$$

The only caution is that now the velocity $\mathbf{u}$ is to be considered the perturbation velocity, that is, the fluid velocity is $\mathbf{U}_{\infty}+\mathbf{u}$ and $\phi$ is the potential of the perturbation velocity only, so that Eq. (2) still holds true. The derivations to follow are thus easily generalizable to the case of uniform, constant velocity away from the wing.

Starting from Eq. (1), we now consider the first term in its rhs, written as, using Eq. (2),

$$
\frac{d}{d t} \int_{C V} \rho \mathbf{u} d^{3} x=\frac{d}{d t} \int_{C V} \rho \nabla \phi d^{3} x,
$$

which, can be written, using Gauss theorem, as

$$
\begin{aligned}
\frac{d}{d t} \int_{C V} \rho \nabla \phi d^{3} x= & \frac{d}{d t} \int_{S_{W}} \rho \Delta \phi \mathbf{n}_{w} d S+\frac{d}{d t} \int_{S_{T}} \rho \phi \mathbf{i} d y d z \\
& -\frac{d}{d t} \int_{S_{\text {body }}} \rho \phi \mathbf{n}_{B} d S,
\end{aligned}
$$

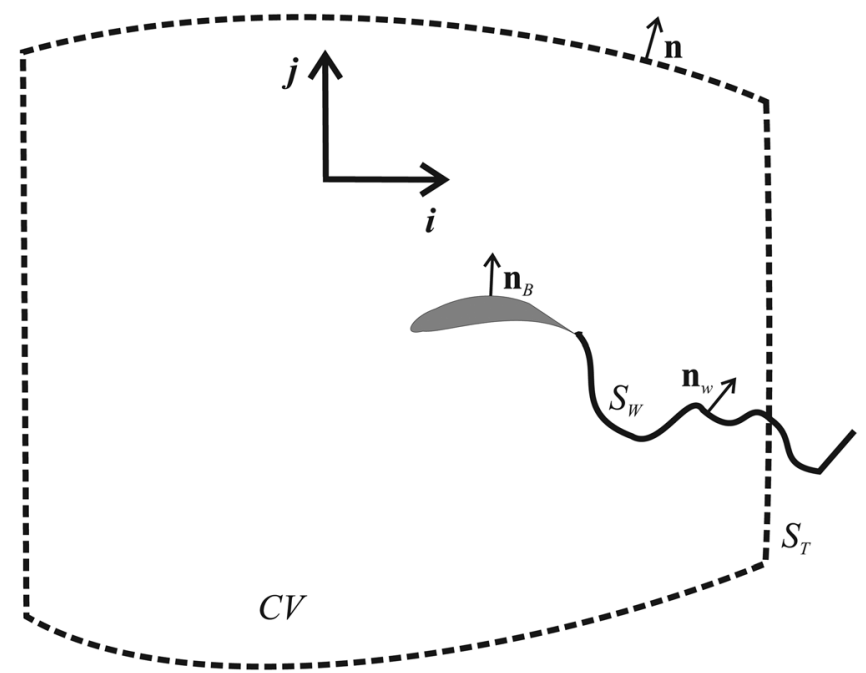

FIG. 1. Sketch of the control volume used (two-dimensional view), denoting also the wake surface $S_{W}$ and the Trefftz plane $S_{T}$. where $\Delta \phi$ is the jump in potential across the wake $\left(\phi_{\text {lower }}-\phi_{\text {upper }}\right), \mathbf{n}_{w}$ is the (upward-pointing) normal to the wake surface $S_{W}$, and the normal to the surface of the body, $\mathbf{n}_{B}$, is that directed toward the outside of the body. The contribution of the surface of $C V$ at infinity to the time derivative of the integral is zero because $\partial \phi / \partial t$ goes to zero faster than $r^{-2}$, with $r$ the distance from the wing (the same argument used in Ref. 7 to derive (1)). To evaluate the second term in Eq. (5), one must consider that $\phi$ has the mentioned jump $\Delta \phi$ across the curve on the Trefftz plane that represents the intersection of this plane with the wake surface. Denoting this curve as $y_{w}(z, t)$, we can write

$$
\begin{aligned}
\frac{d}{d t} \int_{S_{T}} \phi \mathbf{i} d y d z & =\int_{-\infty}^{\infty} \Delta \phi \frac{\partial y_{w}}{\partial t} \mathbf{i} d z+\int_{S_{T}} \frac{\partial \phi}{\partial t} \mathbf{i} d y d z \\
& =\int_{-\infty}^{\infty} \Delta \phi v_{w} \mathbf{i} d z+\int_{S_{T}} \frac{\partial \phi}{\partial t} \mathbf{i} d y d z
\end{aligned}
$$

where $v_{w}(z, t)$ is the vertical velocity of the wake at each $z$ point of the Trefftz plane.

The last term in Eq. (5) can be evaluated using the expression in the appendix of Ref. 5, page 577, $(N$ is the space dimension and $\mathbf{I}$ is the identity tensor)

$$
\begin{aligned}
\int_{S_{\text {body }}} \phi \mathbf{n}_{B} d S= & -\frac{1}{N-1} \int_{S_{\text {body }}} \mathbf{x} \times\left(\mathbf{n}_{B} \times \nabla \phi\right) d S \\
& -\frac{1}{N-1} \int_{S_{\text {body }}} \mathbf{n}_{B} \cdot \nabla \times(\mathbf{x} \times \phi \mathbf{I}) d S .
\end{aligned}
$$

Note that the scalar $\phi$ is not single valued on the surface $S_{\text {body }}$, as $\phi$ has different values at the intersection of the wake with the body, according to the way that intersection is approached, and quantified by the jump $\Delta \phi$ defined above. In this way, denoting by $C_{w}$, a closed curve on the body surface that divides it into two parts and also includes the intersection of the wake with this surface (see the insert in Fig. 2), we can write the last integral in Eq. (7), using Stokes theorem, as

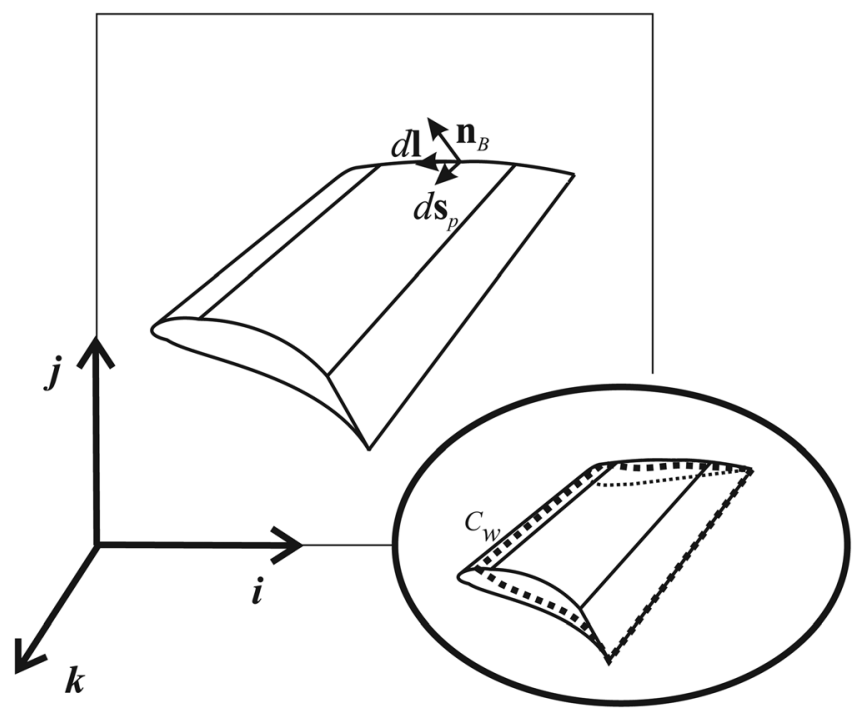

FIG. 2. Intersection of a three-dimensional wing with a vertical plane, denoting also the vectors used to evaluate the wing surface elements. The insert shows, denoted in square symbols, a possible curve $C_{w}$, which divides the wing surface into two surfaces and also runs along the trailing edge. 


$$
\begin{aligned}
\int_{S_{\text {body }}} \mathbf{n}_{B} \cdot \nabla \times(\mathbf{x} \times \phi \mathbf{I}) d S= & \oint_{C_{w}} d \mathbf{s} \cdot\left(\mathbf{x} \times \phi_{\text {upper }} \mathbf{I}\right) \\
& -\oint_{C_{w}} d \mathbf{s} \cdot\left(\mathbf{x} \times \phi_{\text {lower }} \mathbf{I}\right) \\
= & \oint_{C_{w}} \Delta \phi \mathbf{x} \times d \mathbf{s},
\end{aligned}
$$

where the vector $d \mathbf{s}$ is the element of length along the curve, running counterclockwise as observed from above. As before, $\phi_{\text {lower }}$ and $\phi_{\text {upper }}$ denote the values of the velocity potential in the lower and upper side of the curve, respectively, and which can differ from each other only in the zone where $C_{w}$ coincides with the wake, that is, along the trailing edge.

Using Eqs. (2), (7), and (8), it then results

$$
\begin{aligned}
\mathbf{F}_{B} \equiv & \rho \frac{d}{d t} \int_{S_{b o d y}} \rho \phi \mathbf{n}_{B} d S=-\frac{\rho}{N-1} \frac{d}{d t}\left[\oint_{C_{w}} \Delta \phi \mathbf{x} \times d \mathbf{s}\right. \\
& \left.+\int_{S_{b o d y}} \mathbf{x} \times\left(\mathbf{n}_{B} \times \mathbf{u}\right) d S\right] .
\end{aligned}
$$

In this way, with Eqs. (5), (6), and (9), the original expression (1) can be written as

$$
\begin{aligned}
\mathbf{F}(t)= & -\frac{d}{d t} \int_{S_{W}} \rho \Delta \phi \mathbf{n}_{w} d S-\int_{-\infty}^{\infty} \rho \Delta \phi v_{w} \mathbf{i} d z \\
& +\int_{S_{T}} \frac{1}{2} \rho\left(v^{2}+w^{2}-u^{2}\right) \mathbf{i} d y d z \\
& -\int_{S_{T}} \rho(v \mathbf{j}+w \mathbf{k}) u d y d z+\mathbf{F}_{B} .
\end{aligned}
$$

Note that the problematic term proportional to $\partial \phi / \partial t$ has disappeared in Eq. (10) and that this expression is exact within the approximation of inviscid, incompressible fluid and of a thin (massless) wake between the trailing edge and the Trefftz plane.

The first term in the rhs of Eq. (10) can further be evaluated as

$$
\frac{d}{d t} \int_{S_{W}} \rho \Delta \phi \mathbf{n}_{w} d S=\int_{S_{W}} \rho \frac{\partial}{\partial t}\left(\Delta \phi \mathbf{n}_{w}\right) d S+\lim _{\Delta t \rightarrow 0} \frac{\rho}{\Delta t}\left[\int_{S_{W}(t+\Delta t)} \Delta \phi \mathbf{n}_{w} d S-\int_{S_{W}(t)} \Delta \phi \mathbf{n}_{w} d S\right]
$$

where the arguments in the integrals inside the square brackets are evaluated at $t$. The first integral is extended over the upper surface of the wake between the trailing edge and the Trefftz plane, and so it tends to zero as the Trefftz plane gets closer to the trailing edge. Also, the second term can be written as

$$
-\int_{-\infty}^{\infty} \rho \Delta \phi(z, t) u_{t e}(z, t) \frac{\mathbf{n}_{w}}{\mathbf{n}_{w} \cdot \mathbf{j}} d z
$$

where $u_{t e}$ is the horizontal velocity of the trailing edge. This expression can be derived noting that the projection on the horizontal plane of the surface element

$$
\Delta S_{W} \equiv S_{W}(t+\Delta t)-S_{W}(t),
$$

with horizontal span $d z$, must be equal to $d z$ times the horizontal distance advanced by the trailing edge in $\Delta t$, that is

$$
\Delta S_{W} \mathbf{n}_{w} \cdot \mathbf{j}=-u_{t e}(z, t) \Delta t d z
$$

The first term in the rhs of Eq. (10) can thus be computed once the jump of potential across the wake is evaluated form the velocity field as

$$
\Delta \phi(x, z, t)=\int_{-\infty}^{\infty} v(x, y, z, t) d y
$$

Although in principle possible, this evaluation is rather cumbersome and a much more practical expression is obtained if the Trefftz plane could be made uniformly close to the trailing edge, which is possible in near two-dimensional wing geometries. In such a case, due to the closeness of the Trefftz plane to the trailing edge, in addition to the smallness of the first integral in the rhs of Eq. (11), $v_{w}$ in Eq. (10) corresponds to the trailing edge velocity $v_{t e}(z, t)$ at the $z$ point and time considered, and $\Delta \phi(z, t)$ is the wing circulation $\Gamma(z, t)$ at that point. One can thus write a simpler expression for the case of a Trefftz plane very near the trailing edge

$$
\begin{aligned}
\mathbf{F}(t)= & -\int_{-\infty}^{\infty} \rho \Gamma(z, t) v_{t e}(z, t) \mathbf{i} d z \\
& +\int_{-\infty}^{\infty} \rho \Gamma(z, t) u_{t e}(z, t) \frac{\mathbf{n}_{w}}{\mathbf{n}_{w} \cdot \mathbf{j}} d z \\
& +\int_{S_{T}} \frac{1}{2} \rho\left(v^{2}+w^{2}-u^{2}\right) \mathbf{i} d y d z \\
& -\int_{S_{T}} \rho(v \mathbf{j}+w \mathbf{k}) u d y d z+\mathbf{F}_{B} .
\end{aligned}
$$

Note that all the information that is needed to evaluate the terms of the force (13), except $\mathbf{F}_{B}$, is obtained from instantaneous velocity values on the Trefftz plane only, located at $x_{T}$, as $\Gamma(z, t)$ can be evaluated as

$$
\Gamma(z, t)=\int_{-\infty}^{\infty} v\left(x_{T}, y, z, t\right) d y
$$

In this way, a different (stationary) Trefftz plane can be chosen at each instant, so that the condition of closeness to the trailing edge can be satisfied at all times.

The orientation of the wake given by $\mathbf{n}_{w}$ is that at the Trefftz plane and the horizontal and vertical components of the trailing edge velocity, $u_{t e}(z, t)$ and $v_{t e}(z, t)$, respectively, are supposed known form the kinematics of the wing.

For the two-dimensional case, the force per unit span $\mathbf{f}$ is given as 


$$
\begin{aligned}
\mathbf{f}(t)= & -\rho \Gamma(t) v_{t e}(t) \mathbf{i}+\rho \Gamma(t) u_{t e}(t) \frac{\mathbf{n}_{w}}{\mathbf{n}_{w} \cdot \mathbf{j}} \\
& +\int_{S_{T}} \frac{1}{2} \rho\left(v^{2}-u^{2}\right) \mathbf{i} d y-\int_{S_{T}} \rho v u \mathbf{j} d y+\mathbf{f}_{B},
\end{aligned}
$$

with $\mathbf{f}_{B}$ the corresponding expression of $\mathbf{F}_{B}$ per unit span, to be analyzed below.

The term $\mathbf{F}_{B}$, Eq. (9), requires the knowledge of the fluid velocity at successive times at the body surface. A term like this or a similar one appears in all formulations that use control volumes to evaluate forces on bodies. ${ }^{5}$ If no assumption of potential flow had been made, one could evaluate this term from the knowledge of the boundary conditions at the body. However, due to the assumption of potential flow, the velocity entering (9) has to be that closest to the body, but outside the boundary layer.

To evaluate $\mathbf{F}_{B}$, it is convenient to express the body surface element as

$$
\mathbf{n}_{B} d S=d \mathbf{l} \times d \mathbf{s}_{p},
$$

where $d \mathbf{l}$ is the longitudinal element of length on the surface of the body at each $z$ section and $d \mathbf{s}_{p}$ is the element along the span (see Fig. 2). Writing then

$$
\mathbf{n}_{B} \times \mathbf{u} d S=\left(d \mathbf{l} \times d \mathbf{s}_{p}\right) \times \mathbf{u}=(\mathbf{u} \cdot d \mathbf{l}) d \mathbf{s}_{p}-\left(\mathbf{u} \cdot d \mathbf{s}_{p}\right) d \mathbf{l},
$$

we have

$$
\int_{S_{b o d y}} \mathbf{x} \times\left(\mathbf{n}_{B} \times \mathbf{u}\right) d S=\int\left[(\mathbf{u} \cdot d \mathbf{l}) \mathbf{x} \times d \mathbf{s}_{p}-\left(\mathbf{u} \cdot d \mathbf{s}_{p}\right) \mathbf{x} \times d \mathbf{l}\right] .
$$

Also, the jump of potential $\Delta \phi$ on $C_{w}$ is considered to be different than zero only where the wake intersects the body, that is, at the trailing edge, so that $d \mathbf{s}$ in Eq. (9) runs along the span on the trailing edge, but, due to the convention on the circulation used, in the opposite direction to that of $d \mathbf{s}_{p}$, so that ( $\mathbf{x}_{t e}$ is the position of the trailing edge at each $z$-plane)

$$
\begin{aligned}
\oint_{C_{w}} \Delta \phi \mathbf{x} \times d \mathbf{s} & =-\int \Delta \phi \mathbf{x}_{t e} \times d \mathbf{s}_{p} \\
& =-\int \Gamma(z, t) \mathbf{x}_{t e} \times d \mathbf{s}_{p} .
\end{aligned}
$$

With all these considerations, we can write

$$
\begin{aligned}
\mathbf{F}_{B}= & \frac{\rho}{N-1} \frac{d}{d t}\left\{\int \Gamma(z, t) \mathbf{x}_{t e} \times d \mathbf{s}_{p}\right. \\
& \left.-\int\left[(\mathbf{u} \cdot d \mathbf{l}) \mathbf{x} \times d \mathbf{s}_{p}-\left(\mathbf{u} \cdot d \mathbf{s}_{p}\right) \mathbf{x} \times d \mathbf{l}\right]\right\} .
\end{aligned}
$$

Note that $d \mathbf{l}$ has components only on the $z$ plane depicted in Fig. 2, while $d \mathbf{s}_{p}$ may or may not have components on the same plane, but always has a component in the direction $\mathbf{k}$.

In two dimensions, one has $d \mathbf{s}_{p}=d z \mathbf{k}$ and $\mathbf{u} \cdot d \mathbf{s}_{p}=0$, so that the expression of $\mathbf{F}_{B}$ per unit span in the twodimensional case is

$$
\mathbf{f}_{B}=\rho \frac{d}{d t}\left[\Gamma \mathbf{x}_{t e}-\oint \mathbf{x}(\mathbf{u} \cdot d \mathbf{l})\right] \times \mathbf{k}
$$

Although Eq. (10) with Eq. (16) are valid in two and three dimensions, three dimensional velocity data are very difficult to obtain in the full wing span simultaneously, even with multi-plane stereo PIV. ${ }^{13}$ In the case of single-plane stereoscopic PIV, one can obtain the three velocity components on a given plane, as that depicted in Fig. 2, so as to evaluate the integrands in Eqs. (10) and (16) at the given $z$, giving an estimation of the force per unit of $z$ at that particular position. Alternatively, the simpler two-dimensional expression (15) can also be used as an estimator.

There is finally a rougher, but far much simpler approximation for a two-dimensional thin wing, which cannot be precisely proved, but can be justified assuming that the integral in Eq. (17) can be approximated for a thin wing as

$$
\oint \mathbf{x}(\mathbf{u} \cdot d \mathbf{l}) \simeq \Gamma \oint \mathbf{x} d l=\Gamma \mathbf{X}_{C}
$$

where $d l=|d \mathbf{l}|$ and $\mathbf{X}_{C}$ is the body centroid defined as

$$
\mathbf{X}_{C}=\frac{\oint \mathbf{x} d l}{\oint d l} .
$$

The reason for considering a thin wing in Eq. (18) is that for an infinitesimally thin wing, one can write

$$
\oint \mathbf{x}(\mathbf{u} \cdot d \mathbf{l})=\int_{\text {upper }} \mathbf{x}\left[\left(\mathbf{u}_{\text {upper }}-\mathbf{u}_{\text {lower }}\right) \cdot d \mathbf{l}\right],
$$

where $\mathbf{u}_{\text {upper }}$ and $\mathbf{u}_{\text {lower }}$ are the velocities at the upper and lower side of the wing surface at each $\mathbf{x}$ point, and the integral is extended only to the upper part of the surface. In this way, the same components of the velocity that actually contribute to the circulation are those that also have a non zero contribution to the integral in question. The evaluation of $\mathbf{f}_{B}$ then results in

$$
\mathbf{f}_{B} \simeq \rho \frac{d \Gamma}{d t}\left(\mathbf{x}_{t e}-\mathbf{X}_{C}\right) \times \mathbf{k}+\rho \Gamma \mathbf{u}_{t e} \times \mathbf{k}-\rho \Gamma \mathbf{U}_{C} \times \mathbf{k},
$$

where $\mathbf{U}_{C}=d \mathbf{X}_{C} / d t$. Remarkably, the term $\rho \Gamma \mathbf{u}_{t e} \times \mathbf{k}$ cancels exactly the first term in Eq. (15) and also the second one for a close to horizontal wake at the trailing edge $\left(\mathbf{n}_{w} \simeq \mathbf{j}\right)$. Also, for a rapidly flapping wing, one can expect that, roughly, each vectorial component of the term $\rho \Gamma \mathbf{U}_{C} \times \mathbf{k}$ has a similar magnitude to the corresponding one of the two integrals in Eq. (15), but with opposite sign. This can be seen considering that for flapping motions, the largest contributions to the wing circulation, as evaluated in Eq. (14), come from points near the plane of the wing, where the horizontal fluid velocity is close to $U_{C}$, the horizontal component of $\mathbf{U}_{C}$, allowing to write

$$
\int_{S_{T}} \rho v u d y \simeq \rho U_{C} \int_{S_{T}} v d y=\rho \Gamma U_{C}
$$

Similarly, for rapid flapping, with vertical velocities of the trailing edge larger than the horizontal velocity, as is implied for the values of the Strouhal number in propulsive flapping, ${ }^{15}$

$$
\begin{aligned}
\int_{S_{T}} \frac{1}{2} \rho\left(v^{2}-u^{2}\right) d y & \simeq \int_{S_{T}} \frac{1}{2} \rho v^{2} d y \\
& \simeq \frac{1}{2} \rho v_{t e} \int_{S_{T}} v d y \simeq \rho \Gamma V_{C} .
\end{aligned}
$$


The last relation results from considering that the centroid position is roughly half way between the trailing edge and the wing pivoting point (assumed close to the leading edge), so that $V_{C}$, the vertical component of $\mathbf{U}_{C}$, is about $v_{t e} / 2$. Note also that the signs are the correct ones to lead to the approximate cancellation mentioned.

All this results in a very simple final approximation of the force given by

$$
\mathbf{f}(t) \simeq \rho \frac{d \Gamma}{d t}\left(\mathbf{x}_{t e}-\mathbf{X}_{C}\right) \times \mathbf{k},
$$

which requires only the evaluation of integrals like Eq. (14), and can be expected to be applicable with the considerations made, that is, of rapid flapping and pivoting of the wing near the leading edge. Note also that the expression (19) makes sense only if the surviving term in its derivation is important, as expected in the case of rapid flapping, with its continuously changing circulation and resulting non-zero $d \Gamma / d t$.

In order to test (15) and (19), a numerical simulation was performed, using a code that solves the Navier-Stokes equations in two spatial dimensions, in the vorticity-stream function representation, for generic two-dimensional motions of a solid of elliptical cross section. The code employs second-order accurate finite differences, with a fourth-order Runge-Kutta time integration, following the method in Ref. 14, with Thom's formula for the vorticity boundary value, which allows a very efficient use of fast Poisson solvers. Also, the elliptic coordinates that accommodate the solid boundary are transformed to Cartesian coordinates using the same conformal transformation as in Ref. 15, so that a uniform Cartesian grid in the transformed plane results in a nonuniform grid in the real space, with a high resolution near the

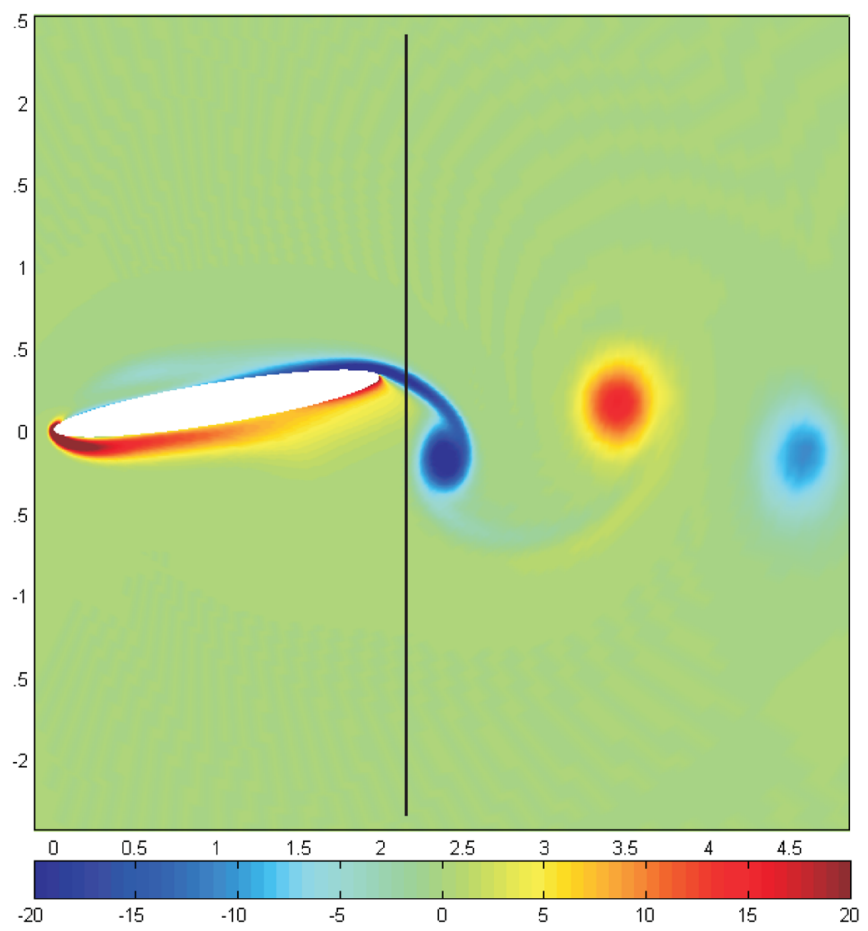

FIG. 3. (Color online) Instantaneous vorticity distribution around a flapping two-dimensional wing of eliptical cross section used in the simulation. Also indicated is one of the Trefftz planes used in the evaluation of the force. body. The boundary condition for the far field stream function is corrected to an exact condition as also done in Ref. 15 with the method developed in Ref. 16. Also following, ${ }^{15}$ pressure forces are evaluated using the flux of vorticity $\omega$ at the solid boundary,

$$
\mathbf{f}(t)=\rho \nu \oint\left(\nabla \omega \cdot \mathbf{n}_{B}\right) \mathbf{x} \times \mathbf{k} d l .
$$

In the case considered, the solid has an elliptical cross section with a ratio of minor to major axis equal to 0.125 . Its leading edge moves with constant horizontal velocity $U_{0}$ to the left, while it oscillates harmonically about this edge with an angular half-amplitude of $0.2 \mathrm{rad}$, giving a maximum vertical velocity of the trailing edge equal to $0.4 \pi U_{0}$. It is worth noting that this configuration is not self consistent, as the motion of the wing is imposed, not resulting from the actual hydrodynamic forces, and is more akin to a wind tunnel experiment as the one studied in Ref. 17.

The Reynolds number based on the leading edge velocity $U_{0}$, chord length $c$, and kinematic viscosity $v$ is equal to 400 , the Strouhal number based on the leading edge velocity, chord length, and flapping frequency $n$ is equal to 1 , while
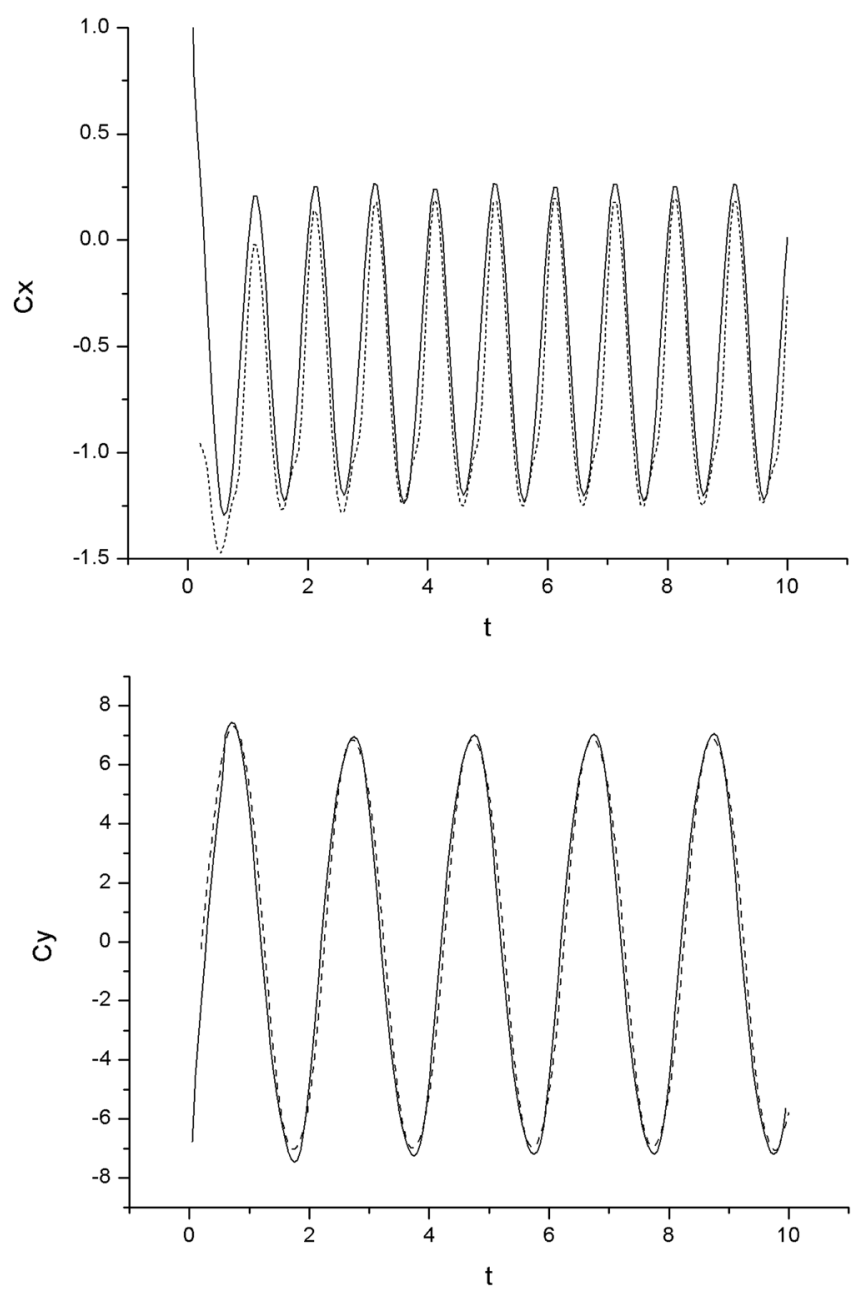

FIG. 4. Non-dimensional horizontal and vertical components of the force on the wing as a function of non-dimensional time. Solid line, numerical simulation and dashed line, formulae (15) and (17). The Trefftz plane is at $1.05 c$ to the right of the leading edge and the surface used for the evaluation of Eq. (17) at $c / \sqrt{\operatorname{Re}}$ from the solid. 
the Strouhal number based on the peak to peak spatial amplitude of the oscillation, $A$, is equal to 0.4

$$
R e=\frac{U_{0} c}{\nu}=400, \quad S t=\frac{n c}{U_{0}}=1, \quad S t a=\frac{n A}{U_{0}}=0.4 .
$$

At this relatively low Reynolds number, with $256 \times 256$ grid points, there is a very good resolution of the boundary layer, with corresponding accurate determination of forces on the solid, and a domain extending five chord lengths in all directions from the wing center. Also, for these values of nondimensional parameters, the average horizontal force is propulsive, in particular, the value of $S t a=0.4$ corresponds to the upper limit of the interval of values of Sta in which flapping propulsive efficiency is maximum. ${ }^{17}$ In the configuration considered, the vorticity is continuously shed at the trailing edge in a thin layer. A snapshot of the vorticity distribution is given in Fig. 3, together with one of the positions of the Trefftz plane employed, which is at $1.05 c$ to the right of the leading edge.

The pressure force on the solid is expressed in nondimensional form in terms of the force coefficients

$$
C_{x, y}=\frac{2 f_{x, y}}{\rho U_{0}^{2} c}
$$
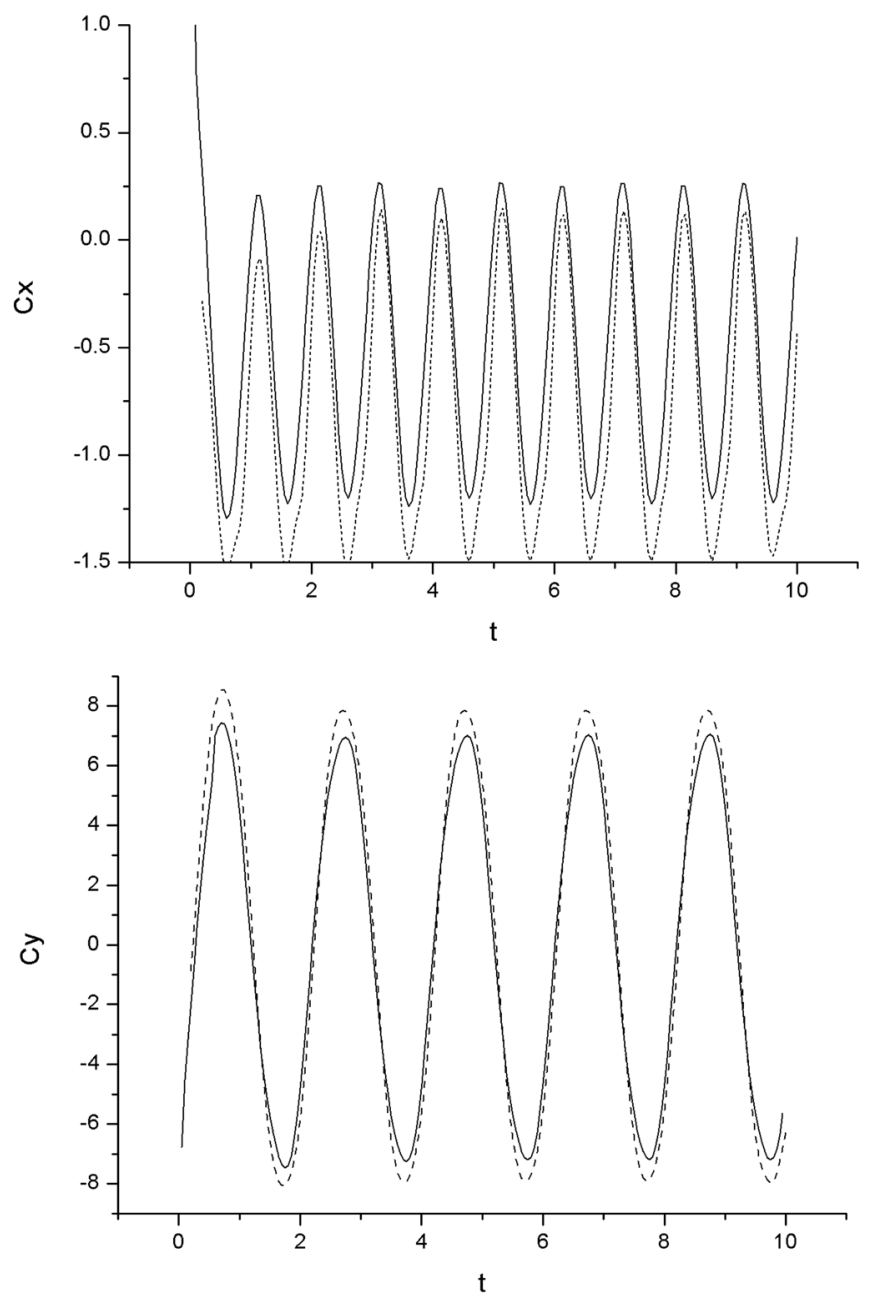

FIG. 5. Non-dimensional horizontal and vertical components of the force on the wing as a function of non-dimensional time. Solid line, numerical simulation and dashed line, formulae (15) and (17). The Trefftz plane is at $1.05 \mathrm{c}$ to the right of the leading edge and the surface used for the evaluation of Eq. (17) at $2 c / \sqrt{R e}$ from the solid. and is shown as the full lines in Fig. 4 as a function of the non-dimensional time

$$
\tilde{t}=\frac{2 U_{0} t}{c} .
$$

The dashed lines in the same Fig. 4 show the force evaluated from Eqs. (15) and (17). The velocity field for this evaluation was taken at fixed intervals $\Delta \tilde{t}=0.05$, and at each time, the Trefftz plane was chosen at $1.05 c$ to the right of the leading edge, as shown in Fig. 3, just outside the viscous boundary layer. The velocity used in the evaluation of the integral in Eq. (17) and of the circulation was that at $c / \sqrt{R e}$ from the solid, an a priori estimation of the boundary layer thickness, which results to be about half the maximum width of the wing. Note that Eq. (17) is independent of the origin of coordinates used, so that no correction for the different positions of the Trefftz plane at each time is needed. The time derivative in Eq. (17) is evaluated at each $\tilde{t}$ as the difference of the magnitude inside the square brackets at $\tilde{t} \pm \Delta \tilde{t}$ divided by $2 \Delta \tilde{t}$. The comparison shows a good agreement between both evaluations of the force at all times.

In order to check the sensitivity to the chosen control surfaces, in Fig. 5, the forces derived from Eqs. (15) and (17)
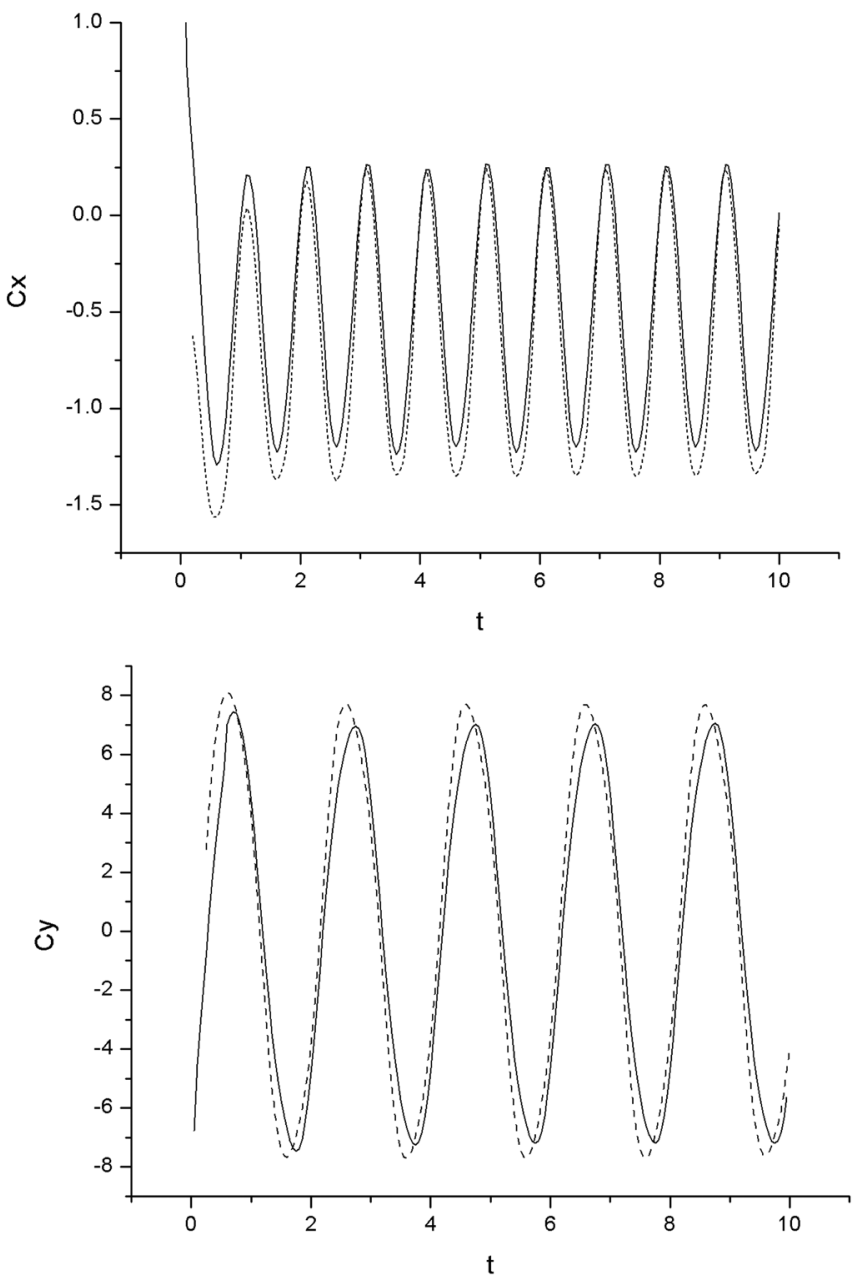

FIG. 6. Non-dimensional horizontal and vertical components of the force on the wing as a function of non-dimensional time. Solid line, numerical simulation and dashed line, formulae (15) and (17). The Trefftz plane is at $1.025 c$ to the right of the leading edge (inside the boundary layer) and the surface used for the evaluation of Eq. (17) at $c / \sqrt{R e}$ from the solid. 
are evaluated using the same Trefftz plane at $1.05 c$ to the right of the leading edge, while the curve for the evaluation of Eq. (17) is taken in this case at $2 c / \sqrt{R e}$, twice the distance used before. Next, in Fig. 6, the Trefftz plane is at $1.025 c$ to the right of the leading edge, while the velocities are again at $c / \sqrt{R e}$ from the surface. These results show that doubling the distance from the surface of the curve used for the evaluation of Eq. (17), going to distances of the order of the wing maximum width, leads to relative errors of about $10 \%$ for $C_{y}$, and of about $20 \%$ for the largest in magnitude values of $C_{x}$, the small positive peaks of $C_{x}$ have errors as large as $50 \%$. On the other hand, letting the Trefftz plane to get too close to the trailing edge, so that the wake is inside the boundary layer, leads to smaller errors, of about $10 \%$ for both components of the force.

Additionally, the results of using the approximation (19) are shown in Fig. 7, using the circulation evaluated as in Eq. (14) for the Trefftz plane at $1.05 c$ to the right of the leading edge. It is interesting that this simple approximation gives such a good estimation of the force, both, in magnitude and phase.

The example so far considered is appropriate to verify the formalism presented as it fulfills the condition for its
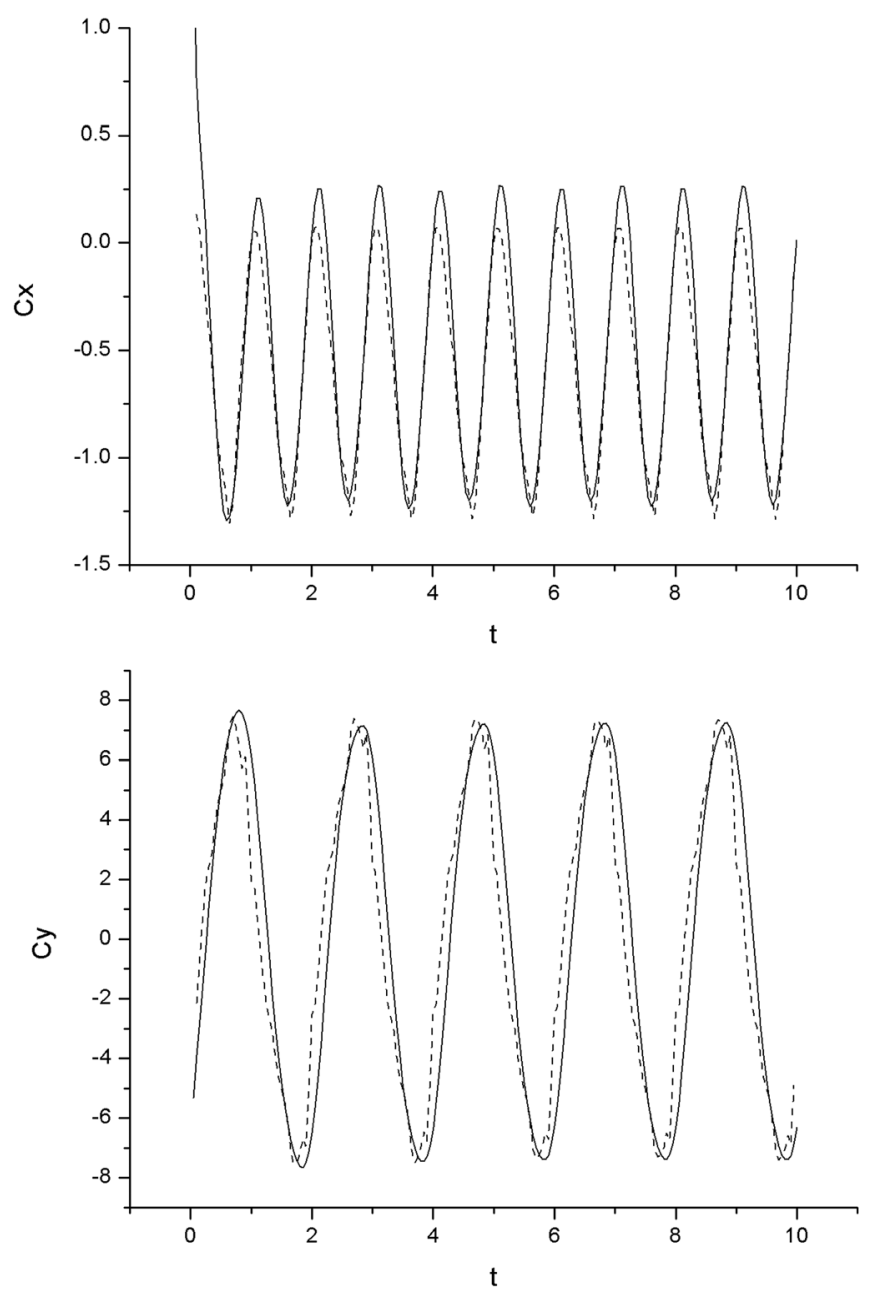

FIG. 7. Non-dimensional horizontal and vertical components of the force on the wing as a function of non-dimensional time. Solid line, numerical simulation and dashed line, formula (19). The Trefftz plane used is at $1.05 \mathrm{c}$ to the right of the leading edge. applicability, that is, the shedding of vorticity in the form of a thin sheet inside the control volume used. In order to see how the formalism performs when this condition is not verified, we now present the same basic configuration, but with different parameters, which are also of interest for the study of flapping motion (the wing dimensions were kept the same in all cases and equal to the previously used ones). The first example of this kind corresponds to a value of the amplitude-based Strouhal number very close to the dragthrust transition, Sta $=0.2 .{ }^{17}$ This number was obtained by halving the frequency previously used, so that the Reynolds number is the same as before, $R e=400$, while the chord length Strouhal number is $S t=0.5$. In this case, the vorticity is continuously shed in the form of a rather thick layer, as shown in Fig. 8.

In Fig. 9 are shown both force coefficients. The thick continuous line corresponds to the numerical solution and the dashed line to the results of the formalism with the Trefftz plane at $1.05 c$ to the right of the leading edge, and the surface used for the evaluation of Eq. (17) at $c / \sqrt{R e}$ from the solid, the same optimal values used in the case with $S t a=0.4$. Also, the result of the simplified expression (19) is shown, in a line with circular symbols. As can be appreciated in this figure, the average thrust is practically zero, corresponding to the thrust-drag transition. The vertical coefficient is rather well reproduced by the full formalism and acceptably good with the approximate expression (19). On the other hand, the horizontal coefficient given by the same formalism predicts a relatively small average thrust and also the peak to peak amplitude is overestimated by about $40 \%$. In contrast, the approximate expression gives a much better estimation.

The next example corresponds to the flapping wing with a stationary leading edge, $U_{0}=0$, akin to a hovering

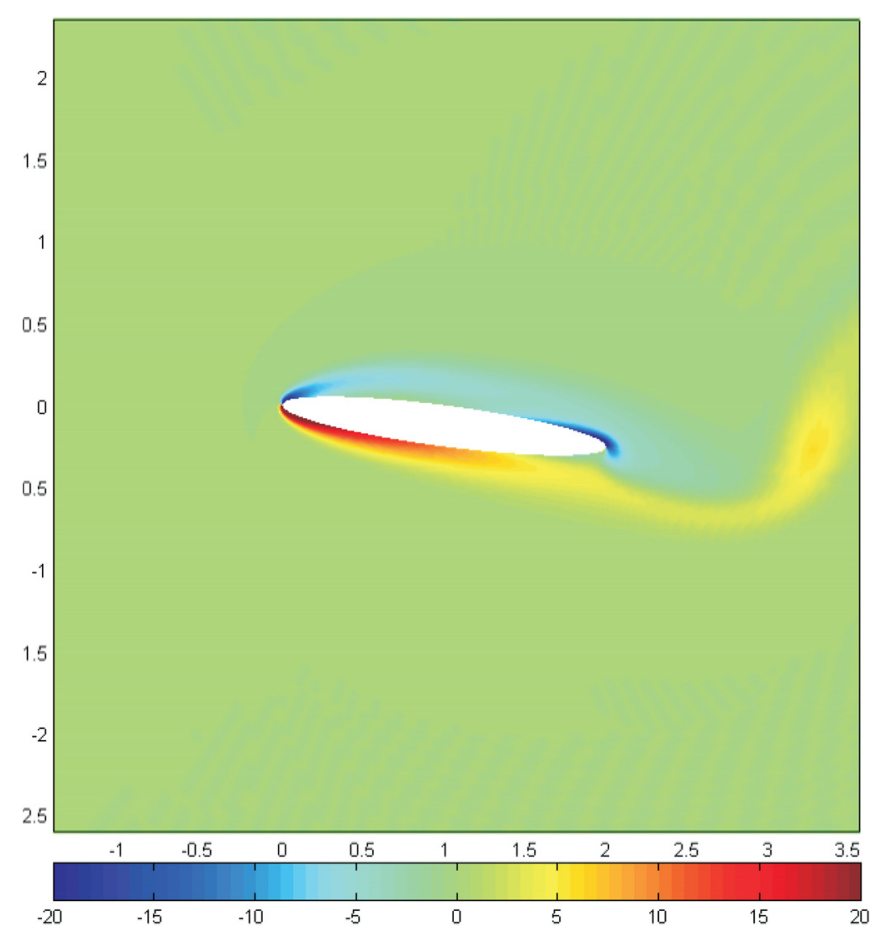

FIG. 8. (Color online) Instantaneous vorticity distribution around the flapping two-dimensional wing for $S t a=0.2$. 

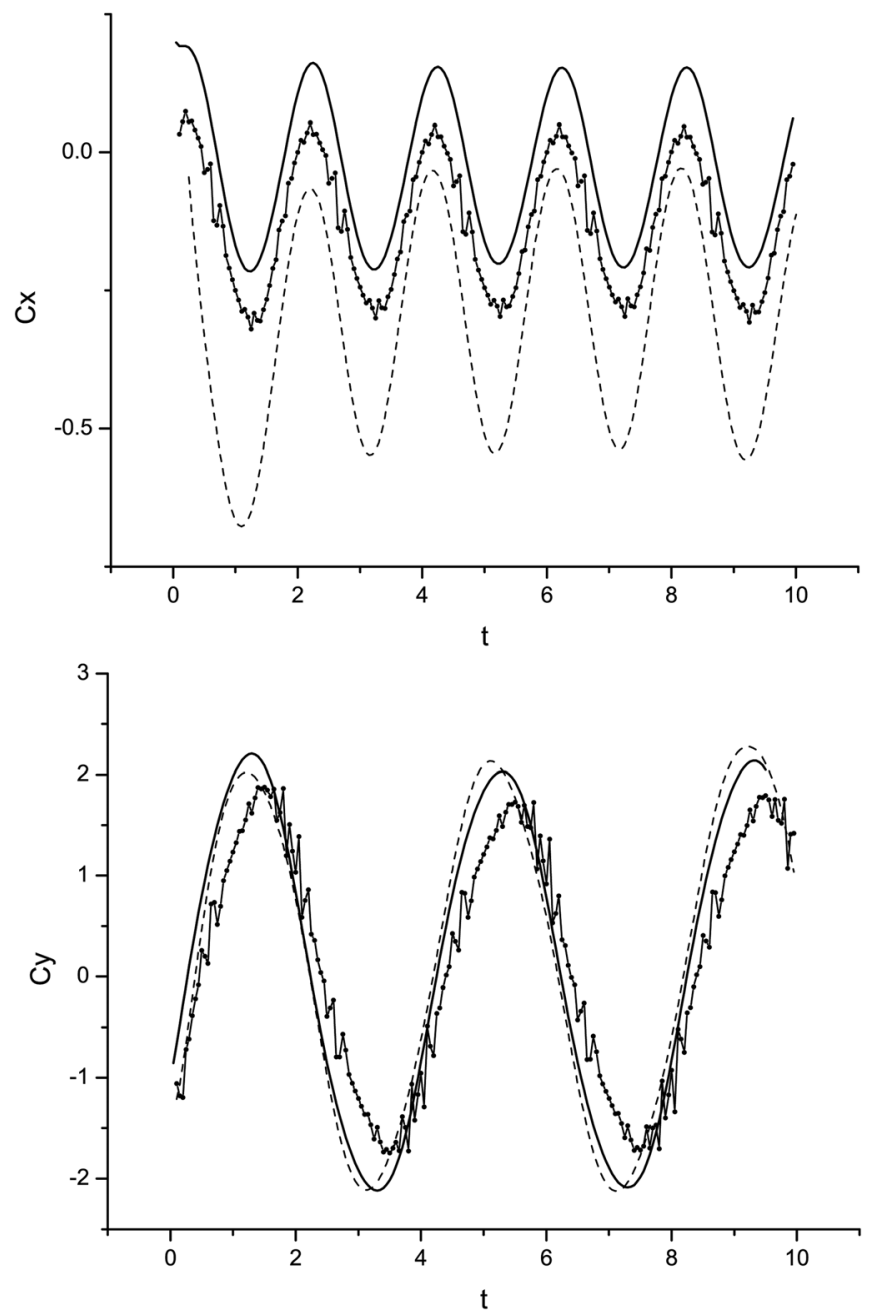

FIG. 9. Non-dimensional horizontal and vertical components of the force on the wing as a function of non-dimensional time for $S t a=0.2$. Solid line, numerical simulation; dashed line, formulae (15) and (17); and line with circular symbols, formula (19). The Trefftz plane used is at $1.05 c$ to the right of the leading edge, and the surface used for the evaluation of Eq. (17) at $c / \sqrt{R e}$ from the solid.

situation. The frequency and amplitude of the oscillatory motion is the same as in the case with Strouhal numbers $S t=1$ and $S t a=0.4$, while the Reynolds number based on the maximum vertical velocity of the trailing edge is $R e=503$. In this case, as can be seen in Fig. 10, the vorticity remains very close to the wing, and inside the control volume, which renders the formalism non applicable in principle. The force coefficients are shown in Fig. 11, with the continuous line representing the numerical simulation, the dashed line the full formalism, and the line with circular symbols the simplified expression (19). Again, the Trefftz plane is at $1.05 c$ to the right of the leading edge and the surface used for the evaluation of Eq. (17) is the same as in the previous case. Also, in order to more easily compare these results with the previous ones, the velocity used to define the force coefficients is the same as in all those cases. As can be seen, similarly to the previous case, the horizontal force coefficient is shifted in average towards negative values and overestimated in peak-to-peak amplitude by also about $40 \%$, while more or less following the correct phase. Remarkably,

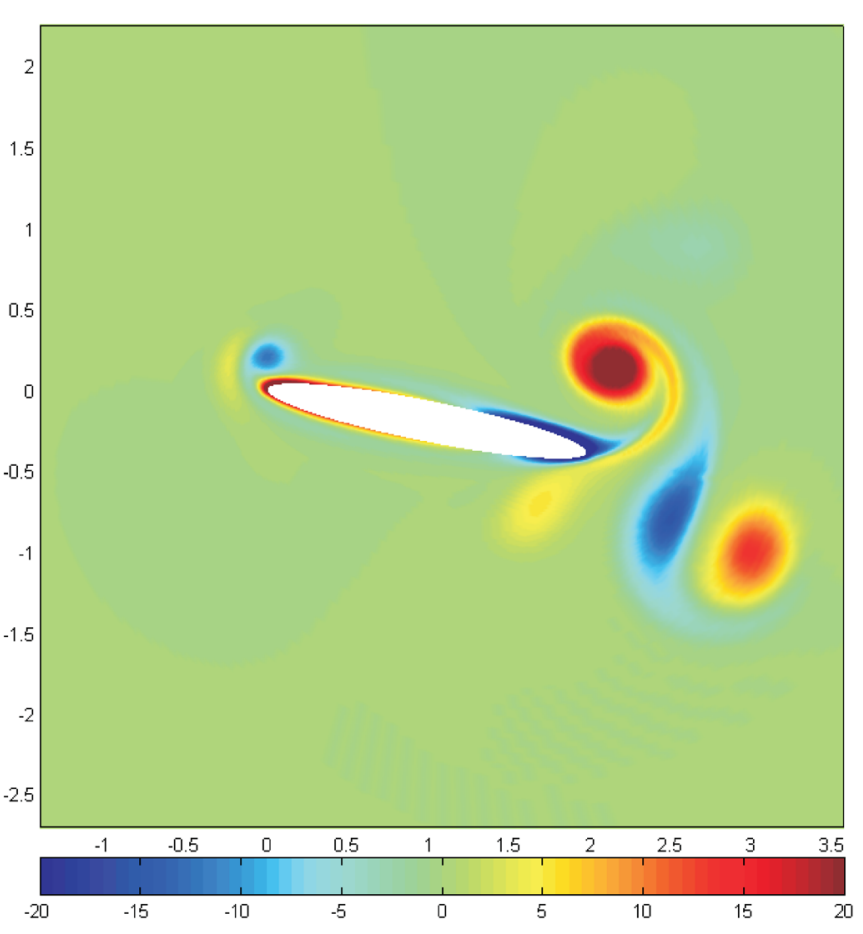

FIG. 10. (Color online) Instantaneous vorticity distribution around the flapping two-dimensional wing with a stationary leading edge.

the simplified expression (19) gives a rather good description of the force in this case. The vertical force coefficient is much better reproduced by the full formalism, while the simplified version gives a less accurate representation of the profile shape, while reasonably representing the amplitude and phase.

Finally, an example is considered with the leading edge moving with the same velocity as in all previous nonstationary cases, and with $S t a=0.4$, in the upper limit of optimal flapping, but with $S t=0.5$. In this way, optimal flapping at half the frequency used before is achieved by doubling the amplitude of the oscillation. As shown in Fig. 12, the vorticity around the trailing edge is again not limited to a thin layer. The force coefficients shown in Fig. 13 indicate a relatively good representation of the horizontal component by the full formalism and a poorer reproduction of the vertical component, specially around the peaks. The simplified version of the formalism is not accurate as to the profiles shape, but gives a reasonable estimation of amplitudes and phases.

In conclusion, expressions for the force on a flapping wing with thin wakes were presented, which can be evaluated from the knowledge of the velocity field in limited regions near the wing. In its practical formulation, with a Trefftz plane uniformly close to the trailing edge, it is applicable to almost two-dimensional wing geometries, so that especially useful is the two-dimensional version of the formalism, which allows the evaluation of the instantaneous force from relatively accessible information of PIV measurements and that further motivates an approximate expression of very simplified form. It is also shown in the cases considered in which the wake is not thin near the trailing edge that the amplitude of the horizontal force tends to be overestimated, and the force itself shifted to give a higher average 

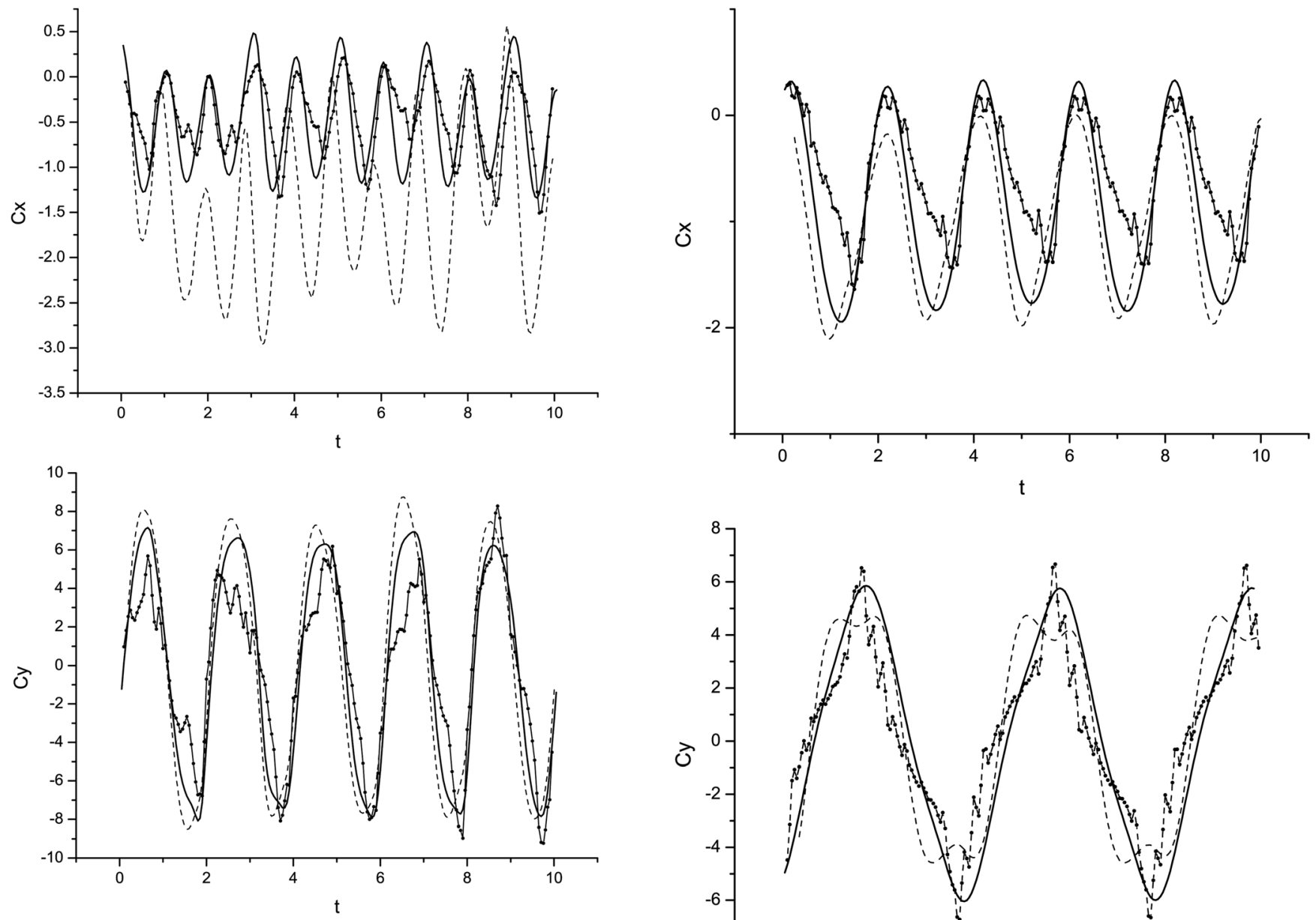

FIG. 11. Non-dimensional horizontal and vertical components of the force on the wing as a function of non-dimensional time for the case with a stationary leading edge. Solid line, numerical simulation; dashed line, formulae (15) and (17); and line with circular symbols, formula (19). The Trefftz plane used is at $1.05 \mathrm{c}$ to the right of the leading edge, and the surface used for the evaluation of Eq. (17) is the same as used in the previous case.

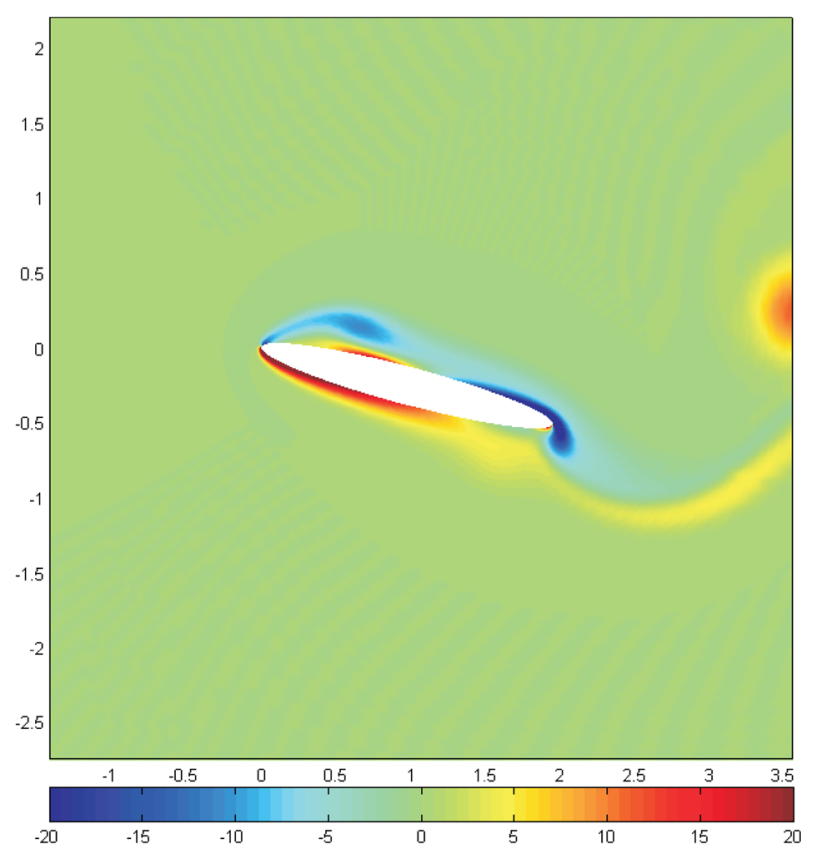

FIG. 12. (Color online) Instantaneous vorticity distribution around the flapping two-dimensional wing for $S t a=0.4$ and $S t=0.5$.

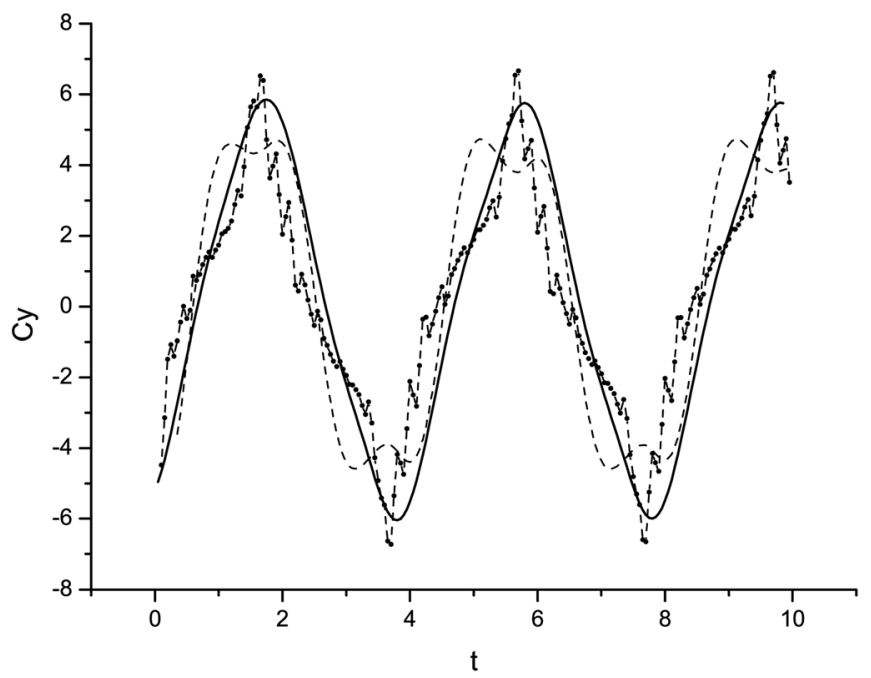

FIG. 13. Non-dimensional horizontal and vertical components of the force on the wing as a function of non-dimensional time for the case with $S t a=0.4$ and $S t=0.5$. Solid line, numerical simulation; dashed line, formulae (15) and (17); and line with circular symbols, formula (19). The Trefftz plane used is at $1.05 c$ to the right of the leading edge, and the surface used for the evaluation of Eq. (17) at $c / \sqrt{\operatorname{Re}}$ from the solid.

thrust. In all cases, however, the simplified version of the formalism gives acceptably good estimations of amplitude and phase of both components of the force. It is possible that the simplified expression (19) is more robust than the original, Eq. (15), because the cancellations that are argued to be verified in the latter are already incorporated in the former relation. However, the simpler expression gives reasonably good results even in situations in which the conditions for the validity of the original formalism are not verified. It would thus be interesting to be able to derive Eq. (19), or a similar one, from a different, more general approach, such as, for instance, the vorticity impulse formulation. ${ }^{3,4}$ Finally, the formalism is easily generalizable to situations with uniform, constant velocity away from the wing as is the case, for instance, in wind-tunnel experiments. In that case, the velocity used in the current expressions must be the perturbation velocity, obtained by substracting from the measured values the uniform velocity, and the full force is obtained by adding the force term (3), which can be evaluated from instantaneous velocity measurements on the Trefftz plane. It is 
interesting to note that this additional term precisely reproduces the Kutta-Joukowski force on a two-dimensional, stationary wing in a uniform flow, with constant wing circulation and without a vortex wake.

${ }^{1}$ Z. J. Wang, "Dissecting insect flight," Ann. Rev. Fluid Mech. 37, 183 (2005).

${ }^{2}$ F. E. Fish and G. V. Lauder, "Pasive and active flow control by swimming fishes and mammals," Ann. Rev. Fluid Mech. 38, 193 (2006)

${ }^{3} \mathrm{~J}$. C. Wu, "Theory for aerodynamic force and moment in viscous flows," AIAA J. 19, 432 (1981)

${ }^{4}$ P. E. Saffman, Vortex dynamics (Cambridge University Press, New York, 1992).

${ }^{5}$ F. Noca, D. Shields, and D. Jeon, "A comparison of methods for evaluating time-dependent fluid dynamic forces on bodies, using only velocity fields and their derivatives," J. Fluids Struct. 13, 551 (1999).

${ }^{6}$ J. Z. Wu, Z. L. Pan, and X. Y. Lu, "Unsteady fluid-dynamic force solely in terms of control-surface integral," Phys. Fluids 17, 098102-1 (2005).

${ }^{7}$ K. C. Hall and S. R. Hall, "Minimum induced power requirements for flapping flight," J. Fluid Mech. 323, 285 (1996).

${ }^{8}$ R. J. Adrian, "Particle-imaging techniques for experimental fluid mechanics," Ann. Rev. Fluid Mech. 23, 261 (1991).
${ }^{9}$ C. Poelma, W. B. Dickson, and M. H. Dickinson, “Time-resolved reconstruction of the full velocity field around a dynamically-scaled flapping wing," Exp. Fluids 41, 213 (2006).

${ }^{10}$ B. Protas, A. Styczek, and A. Nowakowski, "An effective approach to computation of forces in viscous incompressible flows," J. Comput. Phys. 159, 231 (2000).

${ }^{11}$ E. G. Drucker and G. V. Lauder, "Locomotor forces on a swimming fish: Three-dimensional vortex wake dynamics quantified using digital particle image velocimetry," J. Exp. Biol. 202, 2393 (1999).

${ }^{12}$ D. R. Warrick, B. W. Tobalske, and D. R. Powers, "Aerodynamics of the hovering hummingbird," Nature 453, 1094 (2005).

${ }^{13}$ C. J. Kähler, "Investigation of the spatio-temporal flow structure in the buffer region of a turbulent boundary layer by means of multiplane stereo PIV," Exp. Fluids 36, 114 (2004).

${ }^{14}$ E. Weinan and J.-G. Liu, "Vorticity boundary condition and related issues for finite difference schemes," J. Comput. Phys. 124, 368 (1996).

${ }^{15}$ Z. J. Wang, "Vortex shedding and frequency selection in flapping flight," J. Fluid Mech. 410, 323 (2000).

${ }^{16} \mathrm{Z}$. J. Wang, "Efficient implementation of the exact numerical far field boundary condition for Poisson equation on an infinite domain," J.Comput. Phys. 153, 666 (1999).

${ }^{17}$ R. Godoy-Diana, J.-L. Aider, and J. E. Wesfreid, "Transitions in the wake of a flapping foil," Phys. Rev. E 77, 016308-1 (2008). 